\title{
NONTRIVIAL USES OF TRIVIAL RINGS FRED RICHMAN
}

(Communicated by Louis J. Ratliff, Jr.)

\begin{abstract}
Four theorems about commutative rings are proved with the aid of the notion of a trivial ring.
\end{abstract}

0. Introduction. A ring $R$ is trivial if $0=1$ in $R$, that is, if $R$ consists of a single element. Although a trivial ring is a boring object, the fact that a construction results in a trivial ring can be quite interesting. In this note we prove the following four theorems from the point of view of trivial rings; in all four theorems $R \subset T$ are commutative rings with 1 .

(1) If $R^{m}$ maps onto $R^{n}$, and $m<n$, then $R$ is trivial.

(2) If $R^{n}$ maps one-to-one into $R^{m}$, and $m<n$, then $R$ is trivial.

(3) If $\sum a_{i} X^{i}$ is a unit in $R[X]$, then $a_{i}$ is nilpotent for $i \geq 1$.

(4) If $I$ is an ideal in $R[X]$ such that $1 \in T I$, then each element of the annihilator of $I \cap R$ is nilpotent.

Theorem 1 is a standard strong form of the invariance of the rank of a finite-rank free module over a commutative ring [2]; Theorem 2 is a not-so-standard stronger form of the same thing. Alternative formulations presuppose that $R$ is nontrivial and conclude that $m \geq n$; I find (1) and (2) more satisfactory. Theorem 1 says that we can derive the equation $0=1$ from the $n$ equations in $R$ that express the fact that $R^{m}$ maps onto $R^{n}$. Theorem 2 says that we can derive the equation $0=1$ from the conditional equations:

$$
\text { if } \sum_{j=1}^{n} a_{i j} r_{j}=0 \text { for } i=1, \ldots, m, \text { then } r_{j}=0 \text { for } j=1, \ldots, n \text {. }
$$

The techniques developed in this paper were motivated by a desire to prove Theorems 1 through 4 in a constructive manner, in the sense of Errett Bishop [1]. This in fact has been achieved, but the reader need not be familiar with constructive mathematics to follow the proofs. I will mention, however, that by phrasing (1) and (2) in terms of triviality, rather than nontriviality, we don't have to worry about what inequality in $R$ means. In connection with this, the reader might notice that in our proofs we never have to decide whether two elements of $R$ are equal or not (of course we may know that they are equal).

Theorem 3 admits an elegant proof upon observing that each $a_{i}$, with $i \geq 1$, must be in every prime ideal of $R$, and that the intersection of the prime ideals of $R$ consists of the nilpotent elements of $R$. This proof gives no clue as to how to

Received by the editors April 28, 1987.

1980 Mathematics Subject Classification (1985 Revision). Primary 13A99, 03F65; Secondary $13 B 25$. 
calculate $n$ such that $a_{i}^{n}=0$, while such a calculation can be extracted from the proof that we present.

Theorem 4, which is a bit technical, says that if $I$ blows up in $T[X]$, then $I \cap R$ is big; in particular if $I \cap R=0$, and $R$ is nontrivial, then $I$ cannot blow up in $T[X]$. An example of a proper ideal $I$ that blows up in $T[X]$ is obtained by letting $a, b, c$ and $s$ be indeterminates over a field $k$, setting $R=k[a, b, c] /\left(c a, c b, c^{2}\right)$ and $T=R[s] /(s a+(1-s) b)$, and letting $I$ be the ideal in $R[X]$ generated by $1+a X$ and $1+b X$. In this example $I \cap R=(c, a-b)$ has a nonzero annihilator $(c)$, so we cannot strengthen (4) to read that the annihilator of $I \cap R$ is zero.

We rely heavily on the construction of the ring of fractions $S^{-1} R$ for $S$ a subset of $R$ containing 1 and closed under multiplication. Many authors require $S$ not to contain 0 , but this annoying negative statement is unnecessary since we allow $S^{-1} R$ to be trivial. Indeed we draw interesting consequences from the triviality of $S^{-1} R$.

Recall that $S^{-1} R=\{r / s: r \in R$ and $s \in S\}$ with $r_{1} / s_{1}=r_{2} / s_{2}$ if there exists $s$ in $S$ such that $s\left(r_{1} s_{2}-r_{2} s_{1}\right)=0$. In particular, $S^{-1} R$ is trivial if and only if $0 \in S$. We will always choose $S$ to be $\left\{1, s, s^{2}, s^{3}, \ldots\right\}$ for some fixed element $s$ in $R$. This allows us to invert $s$ in $S^{-1} R$, and to conclude that $s$ is nilpotent in $R$ if $S^{-1} R$ is trivial.

1. PROOF OF THEOREM 1. This one is easy; just a warm-up, really. Let $\varphi$ be a map from $R^{m}$ onto $R^{n}$. Because $\varphi$ is onto, there is a map $\psi$ from $R^{n}$ to $R^{m}$ such that $\varphi \psi=1$. Extend $\varphi$ to $R^{n}=R^{m} \oplus R^{n-m}$ by setting $\varphi\left(R^{n-m}\right)=0$, and view $\psi$ as a map into $R^{n}$. If $A$ is the $n \times n$ matrix of $\varphi$, and $B$ the $n \times n$ matrix of $\psi$, then $A B$ is the identity matrix, so $(\operatorname{det} A)(\operatorname{det} B)=1$. On the other hand, $\operatorname{det} B=0$ because the last row of $B$ is all zeros. Hence $0=1$, so $R$ is trivial.

2. PROOF OF THEOREM 2. This proof is more interesting. Let $A$ be the $n \times m$ matrix of a one-to-one map $\varphi$ from $R^{n}$ into $R^{m}$. We first show that the elements in the first column of $A$ are nilpotent. If $r$ is an element in the first column, then let $S=\left\{1, r, r^{2}, \ldots\right\}$ and pass to the $\operatorname{ring} T=S^{-1} R$. It is easily seen that $A$ is the matrix of a one-to-one map from $T^{n}$ into $T^{m}$. Apply elementary row and column operations to $A$, which amounts to changing the bases of $T^{m}$ and $T^{n}$, so that the first column and row of $A$ are 0 except for a 1 in the upper left corner. Let $e_{1}, \ldots, e_{n}$ be the new basis for $T^{n}$. If $m=1$ then $\varphi\left(e_{n}\right)=0$, so $0=1$ in $T$ because $\varphi$ is one-to-one. If $m>1$, then $0=1$ in $T$ by induction applied to the matrix that results from $A$ by deleting the first row and column. Thus $r$ is nilpotent in $R$.

Let $I$ be the ideal in $R$ generated by the elements of the first column of $A$, and let $e_{1}, \ldots, e_{n}$ be the natural basis of $R^{n}$. Then $I^{k}=0$ for some $k$. But if $I^{k} e_{1}=0$ for $k>1$, then $\varphi\left(I^{k-1} e_{1}\right)=0$, so $I^{k-1} e_{1}=0$ as $\varphi$ is one-to-one. Thus $e_{1}=0$ so $R$ is trivial.

3. Proof of Theorem 3. Suppose $f(X)=a_{m} X^{m}+a_{m-1} X^{m-1}+\cdots+a_{0}$ and $g(X)=b_{n} X^{n}+b_{n-1} X^{n-1}+\cdots+b_{0}$ are polynomials in $R[X]$ such that $f(X) g(X)=$ 1. It suffices to show that if $a_{m}, a_{m-1}, \ldots, a_{p+1}$ are nilpotent, and $p>0$, then $a_{p}$ is nilpotent.

Let $S=\left\{1, a_{p}, a_{p}^{2}, \ldots\right\}$ and pass to $S^{-1} R$. We shall show that if $b_{n}, b_{n-1}, \ldots$, $b_{q+1}$ are nilpotent in $S^{-1} R$, and $q \geq 0$, then $b_{q}$ is nilpotent in $S^{-1} R$. This will show that $b_{n}, \ldots, b_{0}$ are nilpotent in $S^{-1} R$, so $1=f(X) g(X)$ is nilpotent in $S^{-1} R$, hence 0 , and therefore $a_{p}$ is nilpotent in $R$. 
So suppose $a_{m}, a_{m-1}, \ldots, a_{p+1}$ and $b_{n}, b_{n-1}, \ldots, b_{q+1}$ are nilpotent in $S^{-1} R$. Because $p>0$ and $f g=1$ we have $\sum_{i+j=p+q} a_{i} b_{j}=0$. Thus $a_{p} b_{q}$ is nilpotent in $S^{-1} R$, so $b_{q}$ is nilpotent in $S^{-1} R$.

4. PROOF OF THEOREM 4. This is the most complicated proof; we break off a piece as a lemma. I am indebted to Steve Merrin for pointing out how to simplify the proof of this lemma.

LEMMA. Let $A$ be a matrix over $R$ such that

(a) if $(r, 0, \ldots, 0)$ is in the row space of $A$ over $R$, then $r=0$,

(b) $(1,0, \ldots, 0)$ is in the row space over $A$ over $T$.

Then $R$ is trivial.

Proof. Designate a finite number of rows of $A$ as good, the rest being designated $b a d$, in such a way that

If a row of $A$ is good, then it contains a 1 , called a $\operatorname{good} 1$, in a column whose other entries are 0 .

To start we may designate all rows as bad. We induct on the number of bad rows. Here we show the constructivist colors: Why not just define a row to be good if it satisfies $(*)$ ? Because to determine, in general, whether or not a row is good, we must be able to decide whether or not an element of $R$ is 1 , or 0 ; and we need not assume the ability to make such decisions for the purposes of proving this lemma. In effect we proceed by backwards induction on the number of known good rows.

Suppose $r$ is in a bad row. Let $S=\left\{1, r, r^{2}, \ldots\right\}$ and consider $A$ as a matrix over $S^{-1} R$. Clearly we can reduce the number of bad rows of $A$ by elementary row operations, so $S^{-1} R=0$ by induction; thus $r$ is nilpotent in $R$. We have shown that all elements in bad rows are nilpotent.

If $\rho_{i}$ denotes row $i$ of $A$, then by hypothesis (b) we have

$$
t_{1} \rho_{1}+t_{2} \rho_{2}+\cdots+t_{m} \rho_{m}=(1,0, \ldots, 0)
$$

for some elements $t_{1}, \ldots, t_{m} \in T$. If all rows are bad, then 1 is nilpotent and we are done. If $\rho_{j}$ has a good 1 in a column other than the first, then $t_{j}=0$. So we may assume that some $\rho_{i}$ has a good 1 in the first column, and the remaining $t_{j} \rho_{j}$ consist of nilpotent elements. Then every entry of $\rho_{i}$ except the first is nilpotent. Let $I$ be the ideal of $R$ generated by these nilpotent elements. If $I^{k}=0$, and $r \in I^{k-1}$, then $r \rho_{i}$ has zero entries except for the first, which is $r$, so $r=0$ by hypothesis (a). Therefore $I=0$, so $\rho_{i}=(1,0, \ldots, 0)$ whereupon $1=0$ by hypothesis (a). Therefore $R$ is trivial.

Returning to Theorem 4, suppose first that $I \cap R=0$. Write 1 as $t_{1} a_{1}+\cdots+$ $t_{m} a_{m}$, with $t_{i} \in T$ and $a_{i} \in I$. Applying the lemma to the matrix of coefficients of the $a_{i}$ we conclude that $R$ is trivial. Now let $I \cap R$ be arbitrary and suppose $r(I \cap R)=0$. Let $S=\left\{1, r, r^{2}, \ldots\right\}$ and pass to $S^{-1} R$. As $I \cap R=0$ in $S^{-1} R$, we conclude that $S^{-1} R$ is trivial, so $r$ is nilpotent in $R$.

\section{REFERENCES}

1. E. Bishop, Foundations of constructive analysis, McGraw-Hill, New York, 1967.

2. P. Cohn, Some remarks on the invariant basis property, Topology 5 (1966), 215-228.

Department of Mathematics, New Mexico state University, las Cruces, NEW MEXICO 88003 